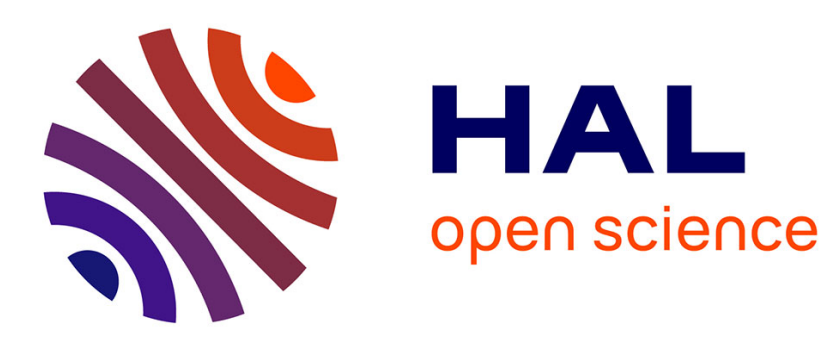

\title{
Modalités de constitution du patrimoine épiscopal de Lucques, VIIIe-Xe siècles
}

\author{
Anne Mailloux
}

\section{To cite this version:}

Anne Mailloux. Modalités de constitution du patrimoine épiscopal de Lucques, VIIIe-Xe siècles. Mélanges de l'École française de Rome - Moyen Âge, 1999, 111 (2), pp.701-723. 10.3406/mefr.1999.3721 . halshs-00505111

\section{HAL Id: halshs-00505111 https://shs.hal.science/halshs-00505111}

Submitted on 6 Feb 2017

HAL is a multi-disciplinary open access archive for the deposit and dissemination of scientific research documents, whether they are published or not. The documents may come from teaching and research institutions in France or abroad, or from public or private research centers.
L'archive ouverte pluridisciplinaire HAL, est destinée au dépôt et à la diffusion de documents scientifiques de niveau recherche, publiés ou non, émanant des établissements d'enseignement et de recherche français ou étrangers, des laboratoires publics ou privés. 


\section{Modalités de constitution du patrimoine épiscopal de Lucques,} VIIle-Xe siècle

\section{Anne Mailloux}

\section{Citer ce document / Cite this document :}

Mailloux Anne. Modalités de constitution du patrimoine épiscopal de Lucques, VIIle-Xe siècle. In: Mélanges de I'Ecole française de Rome. Moyen-Age, tome 111, n². 1999. Résumés des articles. pp. 701-723;

doi : 10.3406/mefr.1999.3721

http://www.persee.fr/doc/mefr_1123-9883_1999_num_111_2_3721

Document généré le 12/06/2016 


\section{MODALITÉS DE CONSTITUTION DU PATRIMOINE ÉPISCOPAL DE LUCQUES, VIII ${ }^{\mathrm{e}}-\mathrm{X}^{\mathrm{e}}$ SIĖCLE}

Le «Diplomatico» de l'Archivio arcivescovile di Lucca contient 1799 actes pour les années 685-1000 (306 pour le VIII ${ }^{\mathrm{e}}$ siècle, 770 pour le IX $^{\text {e }}$ siècle, 726 pour le $X^{\text {e }}$ siècle) ${ }^{1}$. Hansmartin Schwarzmaier ${ }^{2}$ estimait à un cinquième la part des documents relatifs au fonds épiscopal encore conservée. Cette proportion peut toutefois être remise en question par le calcul suivant : le nombre d'actes par notaire, sur l'ensemble de la période, est en moyenne de cinq par an, or certains, au $\mathrm{X}^{\mathrm{e}}$ siècle, sont capables de mettre en forme plusieurs parchemins dans la même journée ou dans un laps de temps très bref ${ }^{3}$. Il est donc probable que la quantité de documents notariaux établis à Lucques pendant le haut Moyen Âge devait être supérieure à cinq fois le total du matériau disponible aujourd'hui. L'échantillonnage est malgré tout suffisant pour établir quelques observations, en tenant compte du fait que ces documents ont été conservés en raison de leur pertinence patrimoniale $^{4}$, et l'on peut considérer qu'ils fournissent un bon observa-

' Cf. graphiques n"1 à 4, "Répartition chronologique des actes conservés». Les actes sont cités ci-après selon leur cote aux archives de l'archevêché ( $\mathrm{AAL}$ ) et/ou leur numéro dans l'édition de D. Bertini, Memorie e documenti per servire alla storia del ducato di Lucca, IV-1 et 2, Lucques, 1818 et 1836 (réimpr. anast. 1994), et D. Barsocchini, Memorie e documenti... di Lucca, V-1 et 2, Lucques, 1837 et 1841 (réimpr. anast. 1971) [abr. "B» suivi de la tomaison et du numéro].

${ }^{2}$ Cf. H. Schwarzmaier, Lucca und das Reich bis zum Ende des 11. Jahrhunderts. Studien zur Sozialstruktur einer Herzogstadt in der Toskana, Tübingen, 1972 (Bibliothek des Deutschen historischen Instituts in Rom, 41).

${ }^{3}$ C'est par exemple le cas d'un certain Petrus notarius domini imperatoris, dont on a conservé sept documents pour une période de neuf jours, en 980 (B, V-3, n"s 1507 à 1513).

${ }^{4}$ Le fonds de Lucques, en effet, n'a pas connu de dégradations ou de destructions accidentelles majeures : les documents détruits ne l'ont donc pas été fortuitement. Il s'agit souvent de pièces devenues inutiles juridiquement. On peut en conclure qu'il existe une distorsion de l'information disponible : les actes conservés sont surtout représentatifs des pratiques patrimoniales de l'évêché et de l'organisa- 
GRAPHIQUE N ${ }^{\circ} 1$. ACTES CONSERVÉS PAR PÉRIODES DE 20 ANS

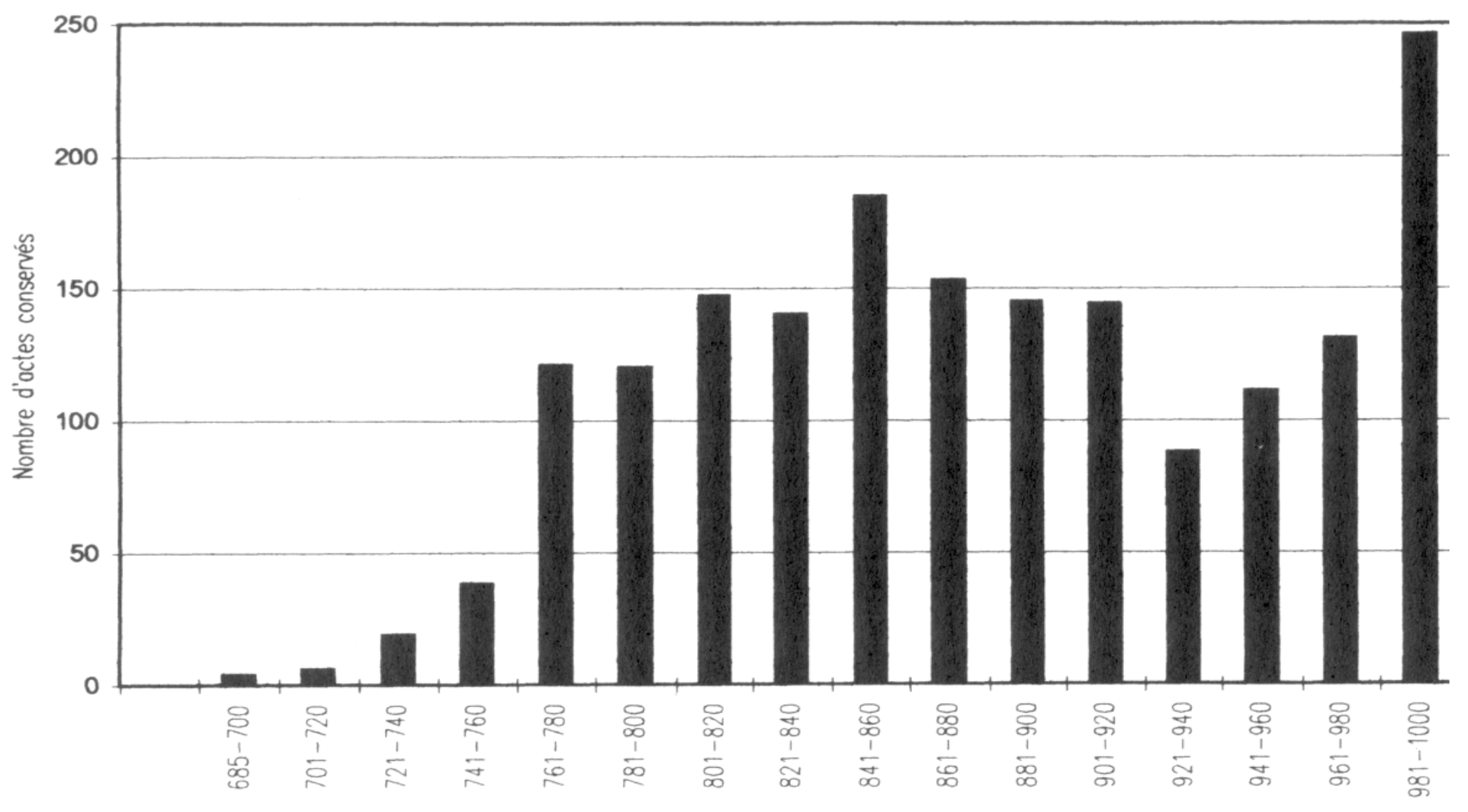

Années d'émission

toire des pratiques patrimoniales de l'institution épiscopale de Lucques : leur cohérence géographique et leur densité permettent une étude des modes de formation et de gestion du patrimoine.

La typologie et la répartition chronologique des actes sont révélatrices de la vie du patrimoine foncier de l'évêché, ainsi que des choix retenus pour le gérer et l'accroître. Les tendances qui ressortent subissent des variations significatives dans le détail. Trois types de documents prédominent, sur les douze recensés dans le fonds : livelli $(54,6 \%)$, ventes $(6,5 \%)$, donations ou offersiones pro anima $(14,2 \%)$ et échanges de biens $(11,2 \%)$. On peut leur adjoindre deux catégories mineures : les promissiones $(1,8 \%)$ et les nominations de prêtres à la tête des églises, plébanes ou non $(6,8 \%)$. Les documents de gestion (brefs et inventaires) sont marginaux $(0,3$ et $0,1 \%$ ).

Deux types de transactions s'imposent dans les mutations de biens-

tion de son domaine. Les dossiers de munimina, essentiellement concentrés au VIII $^{e}$ siècle, ne représentent qu'une faible proportion par rapport à la masse conservée de documents. 
GRAPHIQUE N' 2. RÉPARTITION CHRONOLOGIQUE DES ACTES DU VIII" SIĖCLE

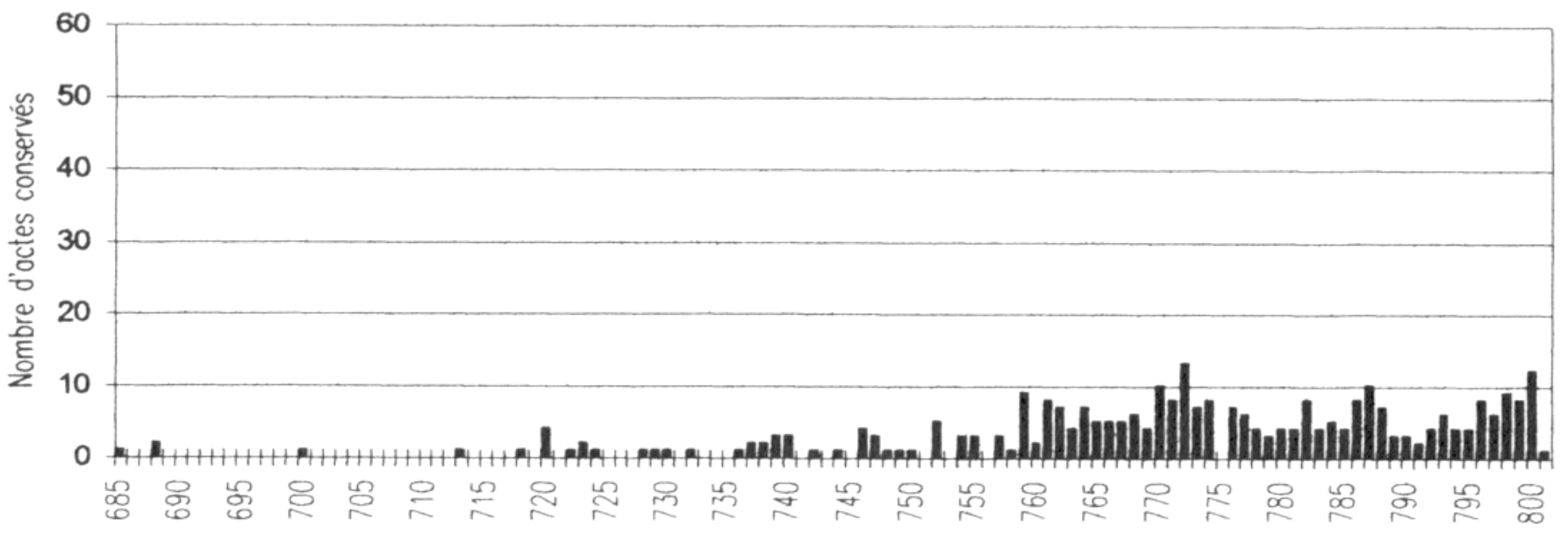

Année d'émission

GRAPHIQUE N' 3. RÉPARTITION CHRONOLOGIQUE DES ACTES DU IX ${ }^{\circ}$ SIÈCLE

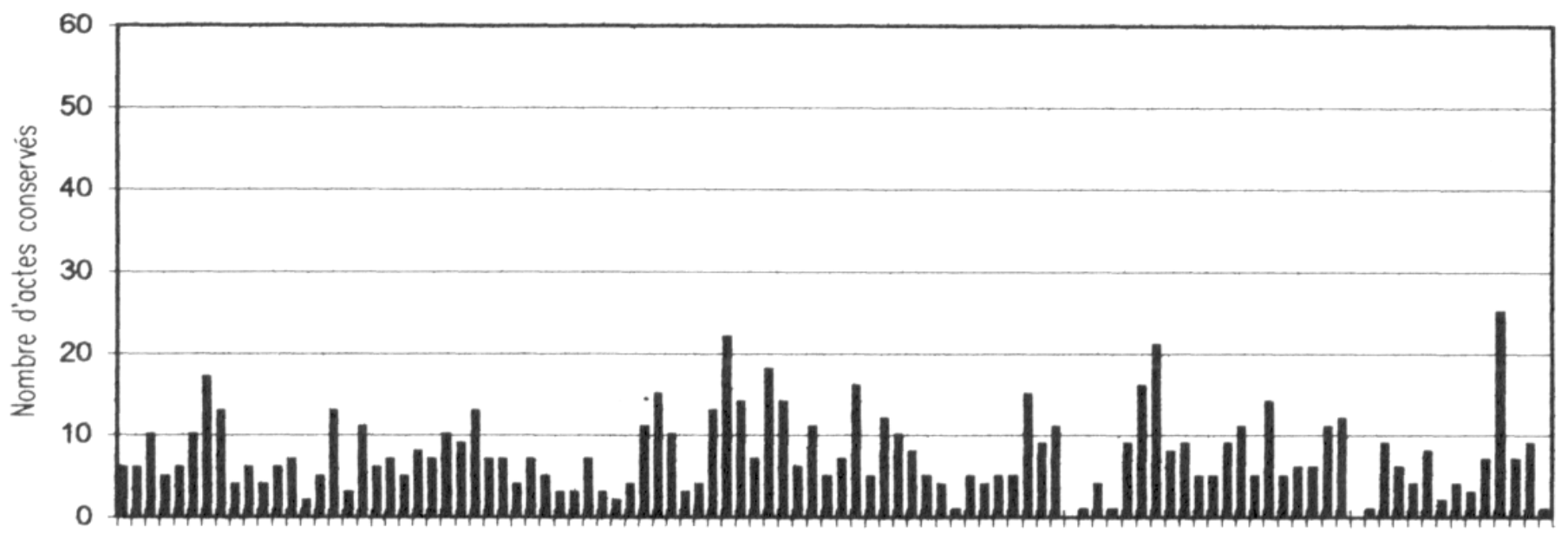

Année d'émission

GRAPHIQUE N* 4. RÉPARTITION CHRONOLOGIQUE DES ACTES DU X* SIÈCLE

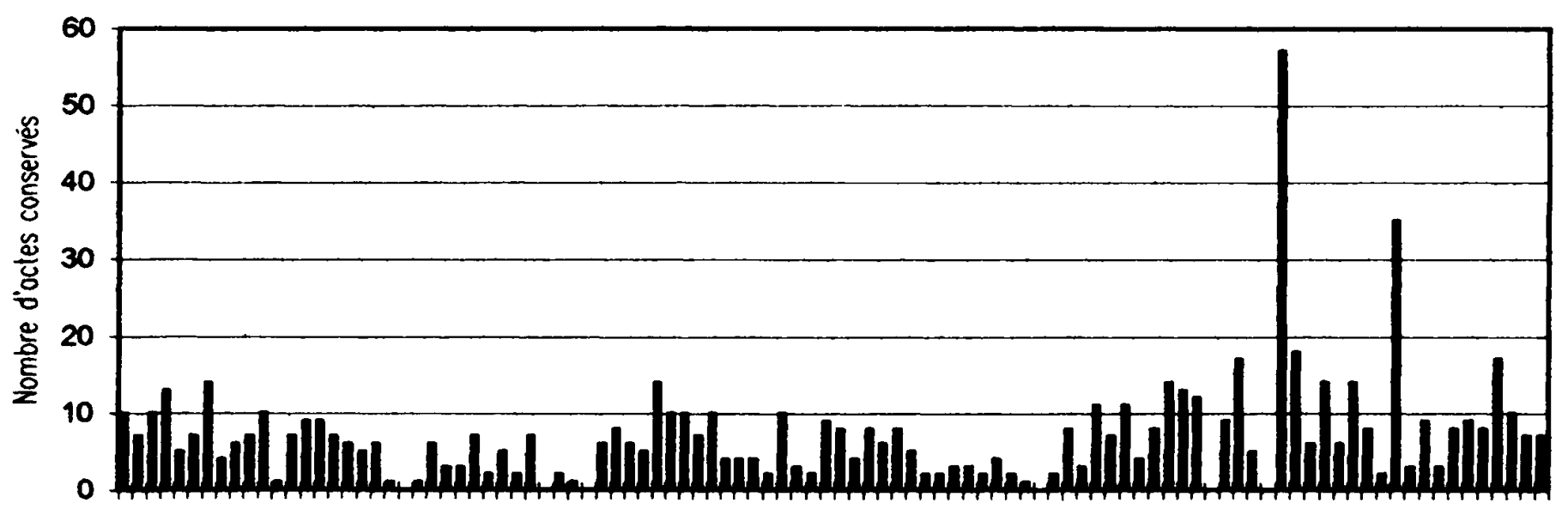

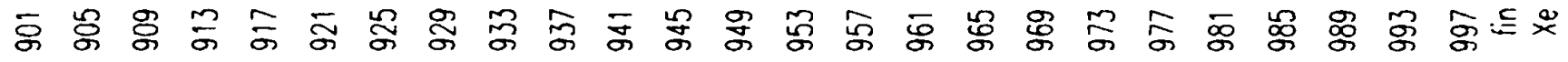


GRAPHIQUE N 5a. RÉPARTITION CHRONOLOGIQUE DES DONATIONS

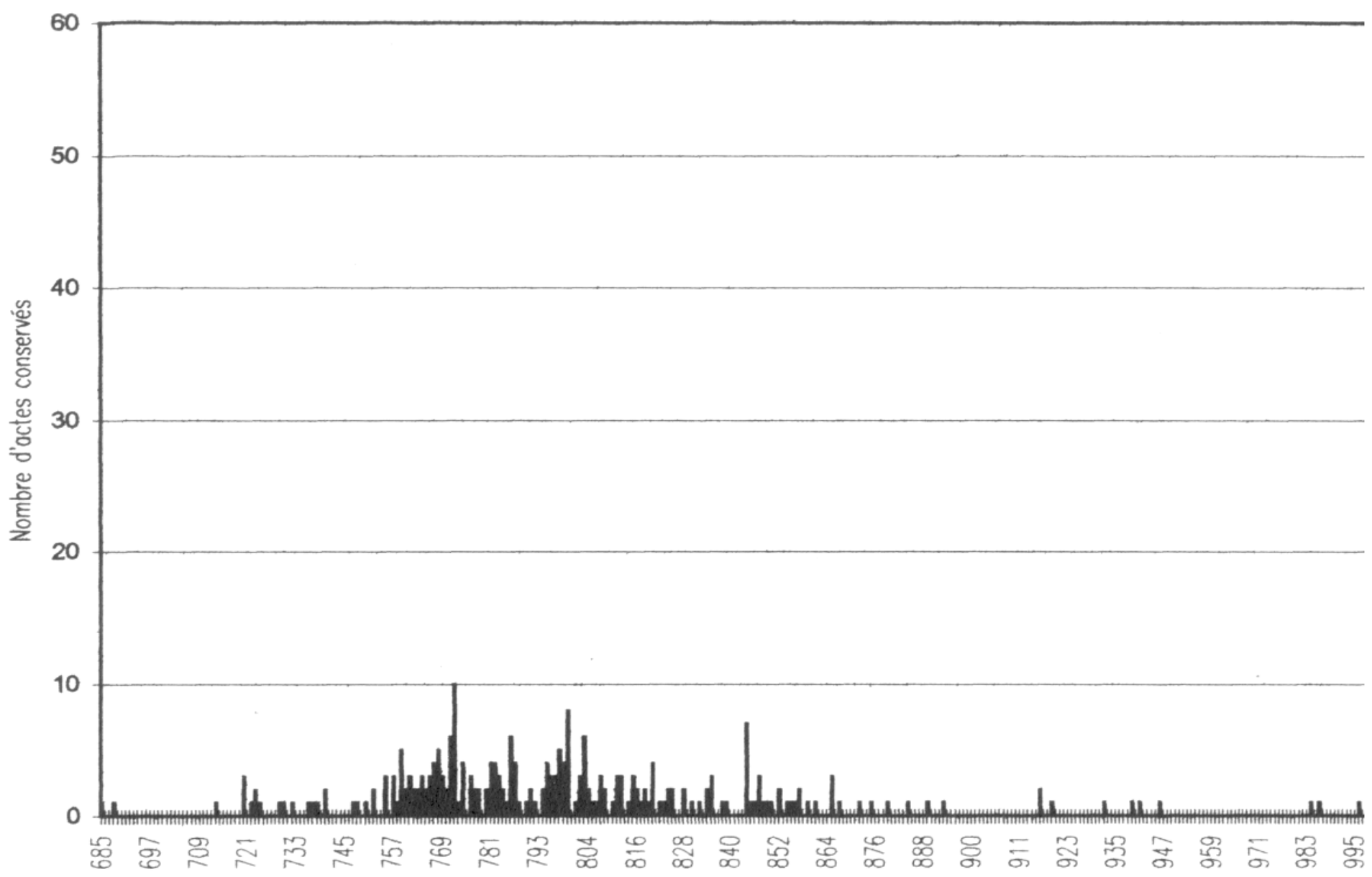

fonds dans la Lucchesia des VIII ${ }^{\mathrm{e}} \mathrm{X}^{\mathrm{e}}$ siècles : les donations et offersiones pro anima, transmissions par héritage, et les livelli.

\section{LES DONATIONS ET OFFERSIONES PRO ANIMA}

Un afflux de biens continu alimente le patrimoine ecclésiastique, enrichi essentiellement par les donations et les exécutions testamentaires, qui sont le principal moyen d'acquisitions foncières pour l'Église. Au VIII $^{e}$ siècle, elles sont majoritaires en pourcentage dans le fonds épiscopal. De 720 à 800, la part de ces donations passe de 63 à 48,3\% , avec une densi-

${ }^{5}$ La répartition typologique des actes fait apparaître une proportion globale de $14,2 \%$ de donations sur l'ensemble de la période. Dans le détail, ces proportions sont les suivantes : $721-740: 63 \%$ des actes conservés; 741-760: 50\%; 761-780: 48,7\%; $781-800: 48,3 \% ; 801-820: 27,2 \% ; 821-840: 12 \% ; 841-860: 12,4 \% ; 861-880: 5,2 \%$. À partir de 881 , la proportion de donations est inférieure ou égale à $2 \%$. 
GRAPHIQUE N $N^{\circ}$ 5b. DONATIONS RÉPARTIES PAR PÉRIODES DE 20 ANS

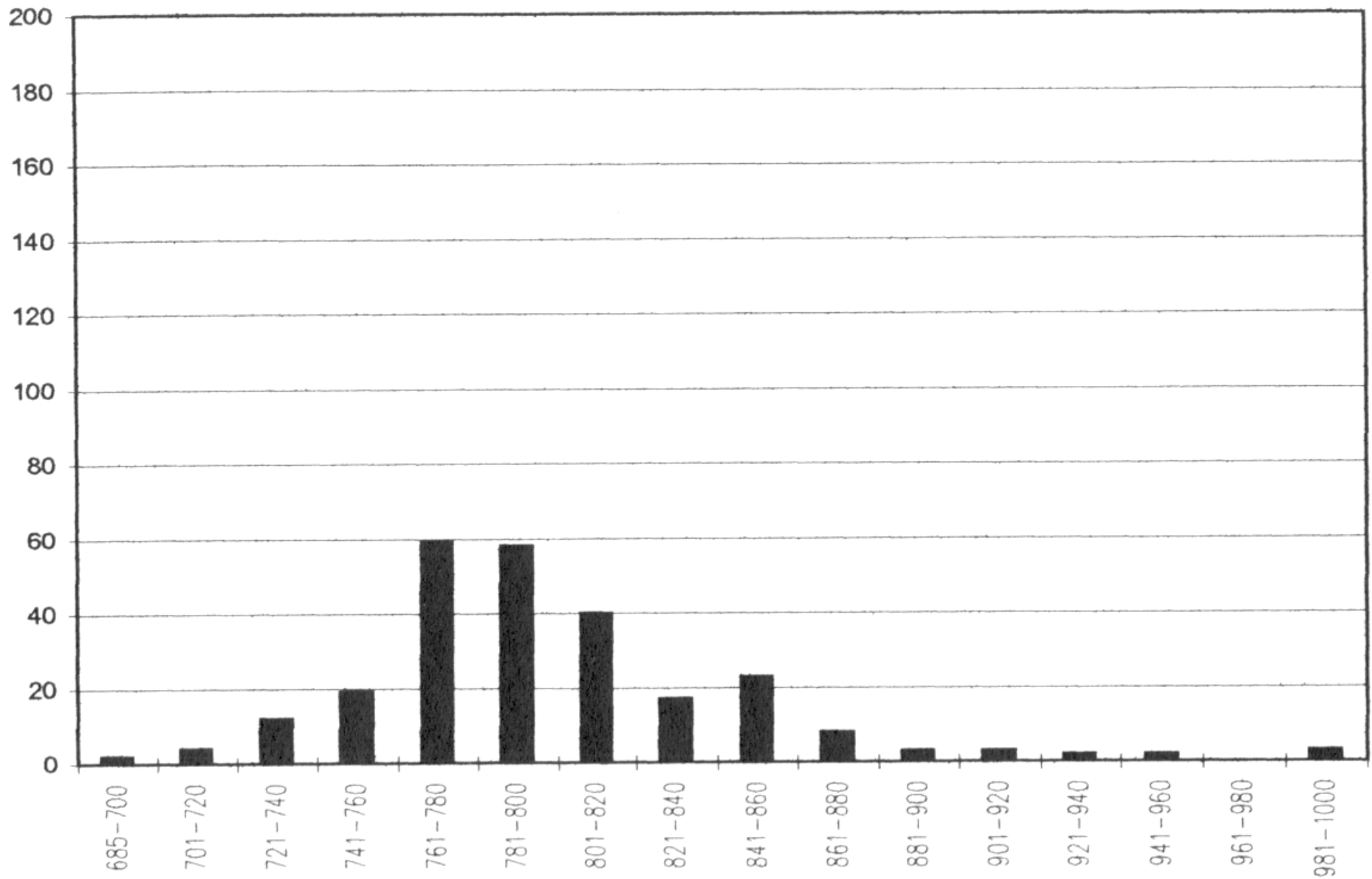

té particulière de 760 à $800^{\circ}$. À partir de 800 , elles sont supplantées par les livelli. Le flux se tarit vers 820 .

Leur répartition chronologique semble correspondre dans un premier temps à l'évolution de la législation au VIII' siècle : elles se multiplient de 750 à 800 , c'est-à-dire dès que les aliénations sont permises par la loi lombarde. Dans l'Édit de Rothari, des obstacles juridiques bloquaient les aliénations et les transferts patrimoniaux. La loi visait à la conservation des biens dans la famille, à l'interdiction de toute transaction décidée par un seul individu au détriment du groupe ${ }^{7}$. Dans le contexte lombard du VII ${ }^{*}$ siècle et du début du VIII ${ }^{\circ}$ siècle, il est très difficile de transmettre des biens à d'autres personnes qu'aux descendants masculins. Si un homme n'avait pas de descendance, on appliquait le procédé de la thingatio, sorte

${ }^{6} \mathrm{Cf}$. graphiques $\mathrm{n}^{\circ \mathrm{5}} 5 \mathrm{a}$ et $\mathrm{b}$, «Répartition chronologique des donations".

${ }^{7}$ Cf. Rothari, 169-175 (édition et traduction par S. Gasparri et C. Azzara, Le leggi dei Longobardi. Storia, memoria e diritto di un popolo germanico, Milan, 1992). 
d'adoption où est désigné un fils légitime qui bénéficie alors des dispositions réservées aux fils légitimes nés du père ${ }^{8}$.

Dans les ajouts à l'Édit de Rothari, on observe une résurgence de la tradition romaine qui s'agrège à la loi lombarde : sous la pression sociale, les normes lombardes s'adaptent à la situation italienne, la législation s'assouplit. L'application des normes devient plus réelle et la législation, de modèle idéal et conservatoire, se met à fonctionner comme une structure dynamique, adaptée aux contraintes sociales et économiques du temps. Au début du VIII ${ }^{e}$ siècle, les modalités de transmission patrimoniales évoluent rapidement, surtout dans les novelles de Liutprand (dès 713) : l'aliénation d'un bien par un seul membre de la famille est rendue possible, ainsi que les donations aux églises, grâce aux dispositions pro anima, moyennant l'accord des personnes tenant le mundium pour les femmes et les enfant mineurs 9 . Le contrôle du patrimoine par les lignages est vite entamé ${ }^{10}$.

Les modifications essentielles touchent les donations à l'église et la transmission des biens féminins. La législation n'est plus contournée par une pseudo-transaction par launegild pour les donations à l'Église. Dans les actes lucquois, la multiplication des donations hors de la sphère familiale ainsi que le grand nombre de donations dominant le tableau des transactions dont bénéficie l'évêché au début du VIII ${ }^{e}$ siècle sont le reflet de cet assouplissement. Le rythme des donations doit donc être relié à la loi : dès que celle-ci les permet, elles se multiplient, et atteignent des proportions écrasantes essentiellement à partir de 750 . On ne peut pour autant évoquer une recrudescence de cette pratique, dans la mesure où il est impossible de déterminer quelle est la tonalité des transactions avant cette date. En effet, la documentation lucquoise se multiplie justement à ce moment parce que la législation de Liutprand modifie le rôle de la charte et le système probatoire"1. Le patrimoine ecclésiastique s'arrondit essentiellement par ces donations.

${ }^{8}$ Un seul cas de ce type est conservé à Lucques, dans une donation de 737.

${ }^{9}$ Cf. Liutprand 6; voir aussi Rothari 153-175, 199; Grimoald 5, Liutprand 1-7, 14, 65, 73, Aistulf 10. Cf. C. Wickham, Economic and social institutions in Northern Toscane in the 8th century, dans Istituzioni ecclesiastiche della Toscana medievale, Galatina, 1980, p. 7-34. Cas d'un enfant donateur: AAL 258 (B, V-2, n 248).

${ }^{10} \mathrm{Cf}$. S. Gasparri et C. Azzara, Le leggi dei Longobardi..., à propos des nouveautés introduites par Liutprand et de l'évolution de la loi lombarde à cette époque. Pour une mise au point récente sur l'absence de testament dans la législation de Liutprand, cf. E. Cortese, Il diritto nella storia medievale. I. L'alto medioevo, Rome, 1995, p. 137-139, avec bibliographie.

"Cf. F. Bougard, La justice dans le royaume d'Italie, de la fin du VIII siècle au début du XI' siècle, Rome, 1995 (BEFAR, 291), p. 70 et suivantes. 
L'absence de véritable testament conduit les personnes devant répartir leur patrimoine à effectuer des dispensationes pro anima, dont le statut juridique semble mal défini : les acteurs donnent leurs biens post mortem à des exécuteurs testamentaires. Une fois le propriétaire décédé, les personnes désignées possèdent pleinement les biens et, si n'était l'obligation morale contractée, peuvent en disposer comme bon leur semble. Cela explique que ces patrimoines sont souvent traités comme le patrimoine propre de l'exécuteur testamentaire. Le plus souvent, celui-ci est un ecclésiastique; le premier exécuteur en mesure de réaliser les volontés du défunt procède à la donation ${ }^{12}$. Cette pratique touche aussi certaines ventes.

On conserve une trace de ces donations grâce à la transmission des munimina après la cession intégrale du bien à l'évêché. À Lucques ont été gardés des petits fonds archivistiques cohérents, enregistrant les transactions de familles dont, pour une raison ou pour une autre, le patrimoine fut ensuite aliéné à l'église. S'il n'est pas toujours possible de déterminer le moment exact où ces biens ont été acquis par l'église, ou placés sous son autorité directe, on peut déterminer un terminus ante quem, en examinant la sédimentation des tituli au dos des actes. Les actes de donation consécutifs à une désignation testamentaire sont explicites sur la procédure : le propriétaire du bien désigne généralement plusieurs personnages chargés de distribuer et de dispenser ses biens pour son salut.

L'ampleur du flux de donations en direction de l'église de Lucques n'est toutefois pas liée au changement de la loi, qui tout au plus encadre une pratique, mais ne la crée pas (la loi s'adapte aux pressions sociales). Elle est à chercher dans le contexte social et politique local. On a longtemps mis ce phénomène sur le compte d'une piété renouvelée de l'aristocratie lombarde, qui marquerait ainsi sa bonne volonté et son adhésion profonde au catholicisme, après sa conversion. Même si cette explication conserve une part de validité, en particulier pour la période suivant immédiatement la conversion (malheureusement très peu documentée, ce qui rend difficile l'appréciation du contexte idéologique et religieux), elle est trop linéaire, et surtout, elle ne permet pas de comprendre pourquoi c'est l'évêché qui draine ainsi le patrimoine aristocratique, et non un monastère, comme c'est le plus souvent le cas.

Or l'examen de la situation de l'évêché au VIII' siècle, maître en partie des destinées de la cité par ses fonctions juridiques et notariales, et plus particulièrement de celle de l'évêque, qui cumule souvent des fonctions re-

${ }^{12}$ Ces donations avec exécuteurs testamentaires s'élèvent à 17. Elles sont réparties comme suit : 2 au VIII ${ }^{e}$ siècle, entre 760 et 774,14 au IX ${ }^{e}$ siècle (essentiellement pendant la première moitié du siècle) et une au $\mathrm{X}^{\mathrm{e}}$ siècle (939). 
ligieuses et politiques, montre combien sont étroits les liens entre l'aristocratie lombarde et l'institution épiscopale. La série des évêques du VIII ${ }^{c}$ siècle et du début du IX" siècle est éloquente : de Walprand à Jacques, tous appartiennent à l'aristocratie locale, parfois de haut rang. Ainsi, Walprand est le fils d'un duc de Lucques ${ }^{13}$ et l'histoire patrimoniale de sa famille témoigne d'une profonde imbrication des pouvoirs épiscopaux et ducaux. Le successeur de Walprand, Peredeo, appartient au même cercle que lui : ils sont semble-t-il liés religieusement, mais aussi par leur famille et leurs biens ${ }^{14}$. Peredeo est aussi important à l'échelle du royaume lombard : il fait partie des "otages-invités" de Charlemagne prélevés parmi les personnages influents du Regnum à l'occasion de la conquête de l'Italie entre 773 et 774 . La haute aristocratie lucquoise, limitée à quelques familles liées entre elles et à la famille royale, tenait et l'évêché et les fonctions ducales.

Le lien organique entre l'élite lombarde et l'évêché est renforcé par la maîtrise des fonctions juridiques et notariales au sein de la cité : au VIII ${ }^{\mathrm{e}}$ siècle, ce sont dans une très large majorité des ecclésiastiques membres de la curie épiscopale qui assument ces deux activités. La formation technique et intellectuelle du notariat à l'époque lombarde est une affaire d'ecclésiastique. Les personnes présentes lors des sessions judiciaires sont aussi des clercs (le plus souvent d'ailleurs les mêmes que nous voyons agir dans les actes de la pratique privée). Enfin, les juges sont fréquemment des ecclésiastiques de haut rang, comme l'évêque qui préside le plus souvent les sessions. Autrement dit, le pouvoir royal lombard s'appuie sur la structure ecclésiastique pour sa représentation à l'échelon local ${ }^{15}$.

${ }^{13}$ Son père, Walpert, fut duc de Lucques entre 713 et 736 . Cf. AAL 8 (a. $722: \mathrm{B}$, V-2, no 10), 17 (B, V-2, n* 18, de 736), 43 (B, V-2, no 44, de 752), 113 (B, V-2, no 111, de 768). Pour l'ensemble de la famille, voir $\mathrm{H}$. Schwarzmaier, Lucca und das Reich..., p. 160-161 et B. Andreolli, Uomini nel Medioevo. Studi sulla società lucchese dei secoli VIII-IX, Bologne, 1983 (Il mondo medievale. Sezione di storia della società, dell'economia e della politica, 4), p. 19-32. La famille Walpert/Walprand est très liée à la cour de Pavie et à la personne royale, cf. AAL 47 (B, IV-1, n" 46, de 754) : Walprand établit un testament avant de se rendre au combat dans l'armée levée par Aistulf.

${ }^{14}$ Cf. H. Schwarzmaier, Lucca und das Reich..., p. 165-166.

${ }^{15}$ Cf. F. Bougard, La justice..., ainsi que H. Keller, Der Gerichtsort in oberitalienischen und toskanischen Städten. Untersuchungen zur Stellung der Stadt im Herrschaftssystem des Regnum Italicum vom 9. bis 11. Jahrhundert, dans Quellen und Forschungen aus italienischen Archiven und Bibliotheken, 49, 1969, p. 1-72, et Id., La Marca di Tuscia fino all'anno Mille, dans Lucca e la Tuscia nell'alto medioevo. Atti del $V$ Congresso internazionale di studi sull'alto medioevo, Spolète, 1973, p. 117-142, pour les structures politiques et la représentation par les évêques du pouvoir royal lombard. Sur le notariat et la justice à Lucques, cf. A. Mailloux, Pratiques notariales et 
Les deux évêques suivants, Jean et Jacques, frères, appartiennent encore au même groupe aristocratique, alors que Charlemagne s'efforce, pendant la même période, de substituer aux cadres politiques et militaires traditionnels des hommes nouveaux. C'est ainsi qu'est nommé le duc Allo, d'origine lombarde mais non lucquoise. Son activité et sa fonction se limitent, dans un premier temps, à la représentation militaire du nouveau pouvoir après le passage des troupes franques dans leur descente vers Rome. Allo assure une transition entre le monde lombard et le monde carolingien : après lui apparaissent, parmi les personnels politiques et juridiques, des Francs.

Cette première période politique correspond, dans les comportements patrimoniaux, à une communauté d'actions et d'appartenance : les donations vont en priorité aux évêques et à leurs représentants, prêtres et recteurs d'église, qui jouent le rôle de relais entre l'aristocratie et les évêques. Le flux des donations en direction de l'évêché ou de ses représentants est solide et régulier.

Les évêques ont dans ce registre le même comportement que les aristocrates laïques : les donations personnelles des évêques du VIII ${ }^{e}$ siècle sont assez fréquentes. Des donations de moindre importance sont faites à certains monastères, comme à San Frediano, d'ailleurs gratifié par l'évêque lui-même. En 838, à l'occasion d'un procès qui met en cause les activités d'un Franc nommé Adelgrim, accusé d'avoir usurpé des biens de l'abbaye sous prétexte qu'ils faisaient partie du fisc, sont enregistrées les déclarations de plusieurs témoins (ecclésiastiques membres de la curie épiscopale, notaires et scabins), prouvant que ces biens appartiennent à San Frediano et relatant les étapes de la genèse de ce patrimoine : au début du $\mathrm{IX}^{\mathrm{c}}$ siècle, l'évêque Jean (780-mars 801) avait donné à son frère Jacques (801-818) son monastère. Jacques le plaça sous l'autorité directe de l'évêché, probablement vers $808-810^{16}$.

Au cours du VIII" siècle, et jusqu'au début du IX'c siècle, la possibilité de faire passer des ensembles fonciers sous la protection de l'évêché tout en en conservant le contrôle patrimonial semble être une des raisons déterminantes de ces donations répétées à l'église de Lucques. Le meilleur exemple est celui du couvent de Santa Maria Ursimanni, situé dans le quart sudouest de la cité. Il fut fondé au début du VIII ${ }^{e}$ siècle par un certain Orso, à

gestion d'un fonds patrimonial à Lucques du VIII au X' siècle. Etude du fonds diplomatique de l'Archivio arcivescovile di Lucca, mémoire de l'École française de Rome, présenté à l'Académie des inscriptions et belles-lettres, 1997.

${ }^{16}$ Cf. AAL 560, avril 838 (= C. Manaresi, I Placiti del "regnum Italiae», I, Rome, 1955 [Fonti per la storia d'Italia, 92], «Inquisitiones», n VI, p. 574-576). 
l'attention de ses filles Ursa et Anselperga ${ }^{17}$. Les abbesses Ursa, Anstruda puis Hiudiperga (au début du IX ${ }^{e}$ siècle) sont issues de la même famille. En 800, Tassilon, fils de Gausprand et petit-fils (ou petit-neveu) d'Orso donne le monastère à la cathédrale de Lucques (Saint-Martin). Puis l'évêché le rétrocède à un membre de la famille et à une abbesse, sous forme de livello. Ainsi, en $852^{18}$, une concession du monastère à Causeramo fils de feu Perifusus et à sa fille Hiudiperga abbesse, contre un cens, rappelle que Causeramo tient ces biens «de jure parentorum meorum». La mise en valeur des biens-fonds est assumée par l'abbesse et sa famille. Ce système, fondé sur une donation et un livello conjoints, est une pratique courante et permet de contrôler les transferts patrimoniaux mettant en jeu des femmes. Si les biens sont propriété de l'évêché, ils sont tenus, exploités et contrôlés par la famille donatrice.

La chronologie des donations s'explique en bonne partie par la modification des comportements de l'aristocratie locale au début du IX ${ }^{\mathrm{e}}$ siècle, liée à l'installation des Francs. Ceux-ci étaient apparus dans les sources vers 790 . Un premier comte/duc, Wichram, est nommé dans un jugement de 797 (gloriosus $d u x$ ), puis en $800(d u x)$ et en 810 (comes; pour la dotation de San Salvatore in Vetroniana) ${ }^{19}$. Cependant, l'appareil juridique et administratif local n'a d'abord connu que peu de changements dans sa forme et ses cadres : les notaires sont encore des ecclésiastiques, et les évêques semblent toujours investis d'une part des pouvoirs publics ${ }^{20}$. Les plaids sont présidés par les missi royaux et par les évêques qui occupent une position dominante dans les structures administratives et juridiques locales.

Le rôle des Francs devient plus net au début du IXe siècle : ce sont les comtes qui procèdent à l'investiture des cadres juridiques locaux, le titre de notarius est alors employé systématiquement (les scribes rappellent systématiquement leur titre dans la rogatio des actes $\left.{ }^{21}\right)$. L'activité juridique et

${ }^{17}$ Pour ce cas particulier, voir le dossier documentaire AAL 8 (B, V-2, $\left.\mathrm{n}^{\circ} 10\right), 24$ (B, V-2, n' 25), 304 (B, V-2, n० 289), 306 (B, IV-1, n' 127), 333 (B, V-2, n 318), 367 (B, V-2, no 351), 432 (B, IV-2, no 18), 452 (B, V-2, no 437), 466 (B, V-2, no 452), 716 (B, IV-2, no 33), 810 (B, V-2, $\left.n^{\circ} 787\right), 828$ (B, V-2, $\left.n^{\circ} 805\right)$. On peut aussi consulter H. Schwarzmaier, Lucca und das Reich..., p. 89.

${ }^{18}$ Cf. AAL 716 (B, IV-2, no 33).

${ }^{19} \mathrm{Cf}$. AAL 271 (B, V-2, $\mathrm{n}^{\circ} 259$, de 797, notitia brevis de jugement en présence du duc); AAL 305 (B, V-2, n' 291, de 800) et 388 (B, V-2, n' 372, de 810), dotation de $\mathrm{S}$. Salvatore in Vetroniana, par Wichram en compagnie de son épouse Mona.

${ }^{20}$ Cf. H. Keller, Das Gerichtsort...

${ }^{21}$ Cf. H. Keller, Das Gerichtsort ..., et Id., La Marca..., ainsi que F. Bougard, La justice..., p. 66-67, sur l'évolution du notariat. Si les notaires de l'époque lombarde ne sont pas investis d'une autorité publique, il ne semble plus que ce soit le cas au 
notariale n'est donc plus une exclusivité du monde ecclésiastique, puisque ces activités sont contrôlées, du moins en partie, par le comte. Cela se traduit par le début de la laïcisation du notariat. Certaines évolutions commencent timidement à se faire jour : ainsi, Wichram emploie un notaire laïc qui semble réserver son activité aux transactions où le comte intervient. Des notaires comtaux existent, certains semblent aussi se spécialiser dans le service des vassaux originaires du nord des Alpes. Entre 803 et 815 , un groupe d'origine franque s'impose dans les souscriptions. Jusque vers 815 à peu près, la maîtrise des structures juridiques et notariales locales n'échappe pas encore au contrôle de l'aristocratie lombarde et de l'évêché (des notaires ecclésiastiques subsistent jusqu'à cette date), mais la politique carolingienne consiste à instiller progressivement des modifications dans l'organisation institutionnelle du Regnum et à mettre en place de nouveaux cadres politiques et administratifs.

On observe par la suite un basculement : au cours des années 815 à 847 , le personnel juridique et notarial ecclésiastique s'efface. Les ecclésiastiques, jusque-là en charge de la gestion des affaires judiciaires, disparaissent de cet espace, à l'exception de l'évêque qui siège toujours au plaid. Le nombre de notaires ecclésiastiques diminue considérablement, jusqu'à devenir résiduel et disparaître enfin au milieu du IX ${ }^{*}$ siècle, tandis que le personnel judiciaire (lociservatores, puis scabini) se laïcise au cours de la même période : en 809 intervient le dernier lociservator diacre, et il n'y a plus de scabini ecclésiastiques dans les plaids de $813,815,822,840^{22}$. L'activité des scabins ecclésiastiques est circonscrite à quelques mentions dans les actes privés, puis ils disparaissent définitivement en tant que tels en 847. Au même moment se met en place un duché fort sous l'autorité de la famille des Boniface, investis d'une puissance politique et militaire qui dépasse la zone d'influence lucano-pisane. Boniface ${ }^{\text {er }}$, d'origine bavaroise, est cité pour la première fois en 812-813. Il entraîne dans son sillage des Francs, Alamans et Bavarois (en moindre nombre), qui occupent progressivement une part des fonctions de la cité de Lucques, principalement dans les cours de justice. L'évolution des institutions publiques favorise un contrôle accru des comtes et ducs originaires du nord des Alpes sur le territoire de Lucques et sur les activités patrimoniales : les comtes détenant les rênes du pouvoir juridique, ils contrôlent les activités notariales, ne seraitce que parce que l'autorité des notaires doit être validée par la puissance

début de la période carolingienne : l'attention portée par les souverains carolingiens au notariat, dès la fin du VIII ${ }^{c}$ siècle, montre que l'institution est l'objet d'un soin tout particulier.

${ }^{22}$ C. Manaresi, I Placiti..., ns 26, 29, 33, 44. 
qu'ils incarnent. Ce contrôle va encore plus loin : ainsi, le comte envoie des missi pour vérifier l'équité et la conformité aux normes des transactions foncières, notamment des échanges de biens. Les comtes de Lucques exercent ainsi un droit de regard direct sur les pratiques patrimoniales de l'aristocratie lucquoise d'origine lombarde et des évêques. À la même époque enfin, les évêques ne sont plus exclusivement issus de l'aristocratie lombarde lucquoise, mais d'origine franque, comme Bérenger (834-843) et Ambroise (843-852) ${ }^{23}$, ce qui accroît la marge de manœuvre du comte.

Dans ce contexte politique en mouvement, l'aristocratie d'origine lombarde établie à Lucques tend à préserver son patrimoine contre l'installation des Francs : la donation à l'évêque, membre du même groupe aristocratique, sert de refuge foncier et politique. Ainsi, les donations ont encore un bon niveau entre 801 et 820 . À cette époque, les deux évêques successifs, Jacques (801-818) et Pierre $I^{\text {er }}$ (818-834), sont d'origine locale. Les donations à l'évêché et l'investissement dans des charges ecclésiastiques permettent à ces Lombards de maintenir leur position sociale et de limiter les effets de l'arrivée des Francs et la confiscation des pouvoirs publics par les nouveaux cadres mis en place une vingtaine d'années après la conquête. Le nombre d'ordinations à la tête des églises est très élevé entre 800 et 820 , et les livelli augmentent au même moment : le mouvement de donation-rétrocession peut s'entendre comme un ajustement lié à l'arrivée des Francs.

Le mécanisme des donations semble donc révéler des liens étroits entre les familles lombardes et l'évêché, tant que celui-ci recrute chez elles : le réflexe de soutien est sous-tendu par l'éminence politique et administrative de l'évêque pendant la période lombarde. Les relations se tendent au cours du IX ${ }^{\mathrm{e}}$ siècle, approximativement à partir de 820 : les structures institutionnelles traditionnelles se délitent et les mécanismes patrimoniaux, les stratégies de l'évêque changent du tout au tout. Cela explique peut-être l'entrée dans une phase défensive de gestion du patrimoine, qui se traduit essentiellement par la progression des concessions livellaires : il s'agit pour l'évêque de compenser sa perte d'influence politique par la création d'un ré-

${ }^{23}$ Cf. H. Schwarzmaier, Lucca und das Reich..., p. 92-95. Bérenger apparaît pour la première fois dans les actes en 837 (AAL 555; B, V-2, n' 535). Il est présent dans les sources jusqu'au 12 juin 843 (AAL 610; B, V-2, n' 587). Son successeur Ambroise apparaît le 15 décembre de la même année (AAL 616; B, V-2, n' 593). L'analyse prosopographique permet de le rattacher à une famille de la noblesse impériale. Il meurt après le 29 février 852 (AAL 715; B, V-2, n' 691), mais son successeur Jérémie n'apparaît qu'au mois de décembre (AAL 716; B, IV-2, $n^{\circ} 33$ ), ce qui laisse une certaine marge d'imprécision dans la chronologie. La courbe chronologique des actes conservés connaît d'ailleurs à cette date un fléchissement qui traduit probablement une période politique trouble et un arrêt des affaires courantes. 
seau de clientèle. Mais lorsque l'évêché cesse de contrôler les institutions relatives à la maîtrise de la terre (notariat et fonction juridique), ce système n'est plus une solution de repli patrimonial. Lorsque l'appareil politique et ecclésiastique est pris d'assaut et tenu par les Francs, les Lucquois n'ont plus intérêt à alimenter le patrimoine épiscopal : cela représenterait une perte de contrôle de leur espace. Le tarissement des donations, à partir de 820, s'explique probablement en partie par ce critère. La courbe des donations présente une nette diminution lorsque des personnalités d'origine franque - étrangères en tout cas au monde traditionnel lucquois - arrivent à la tête du diocèse (Bérenger, 834-843; Ambroise, 843-852). Il n'y a alors quasiment plus aucune donation à l'évêché.

En même temps que décline le nombre des donations augmente celui des concessions livellaires. La phase de constitution du patrimoine a laissé place à celle de la gestion, avec ses conséquences, dont l'entrée de plainpied de l'évêché dans un jeu de type clientéliste. L'évêché crée une clientèle grâce aux livelli et établit des liens privilégiés avec l'aristocratie, dont il est le principal interlocuteur foncier. Cette stratégie n'est possible que par le rassemblement, au VIII' siècle, d'un patrimoine important essentiellement issu de donations. Vers 800-820, l'augmentation des livelli semble s'expliquer par le jeu de donation-rétrocession, mené par l'évêque - encore lombard - pour maintenir son influence réelle au niveau du territoire lucquois. Par la suite, les évêques Ambroise et Bérenger (tous deux "allogènes») mettent en place une politique concessionnaire fondée uniquement sur les livelli, contrats emphytéotiques non agricoles, pour créer un réseau de soutien qui s'avère toutefois assez instable.

\section{LES LIVELLI}

Entre 800 et 820 , l'augmentation spectaculaire des concessions livellaires dans le tableau patrimonial lucquois est l'élément marquant de l'histoire des transactions foncières épiscopales. Pour les trois siècles étudiés, les livelli représentent $54,6 \%$ des actes conservés ${ }^{24}$. Surtout, si les ventes sont établies régulièrement, et sensiblement dans la même proportion jusqu'en 820 , les donations s'écroulent, tandis que les concessions livellaires augmentent de façon spectaculaire au même moment.

${ }^{24} \mathrm{Cf}$. graphiques 6 a et $\mathrm{b}$, Répartition chronologique des livelli. La proportion des livelli dans la typologie des actes conservés se répartit des la façon suivante : 721 $740: 53 \% ; 741-760: 5,2 \% ; 761-780: 7,4 \% ; 781-800: 15 \% ; 801-820: 32,6 \% ; 821-840$ : $57,8 \% ; 841-860: 61 \% ; 861-880: 66,6 \% ; 881-900: 72 \% ; 901-920: 77 \% ; 921-940$ : $64,7 \% ; 941-960: 54,9 \% ; 961-980: 57,2 \% ; 981-1000: 80,8 \%$. 
GRAPHIQUE No 6a. RÉPARTITION CHRONOLOGIQUE DES LIVELLI PAR ANNÉS

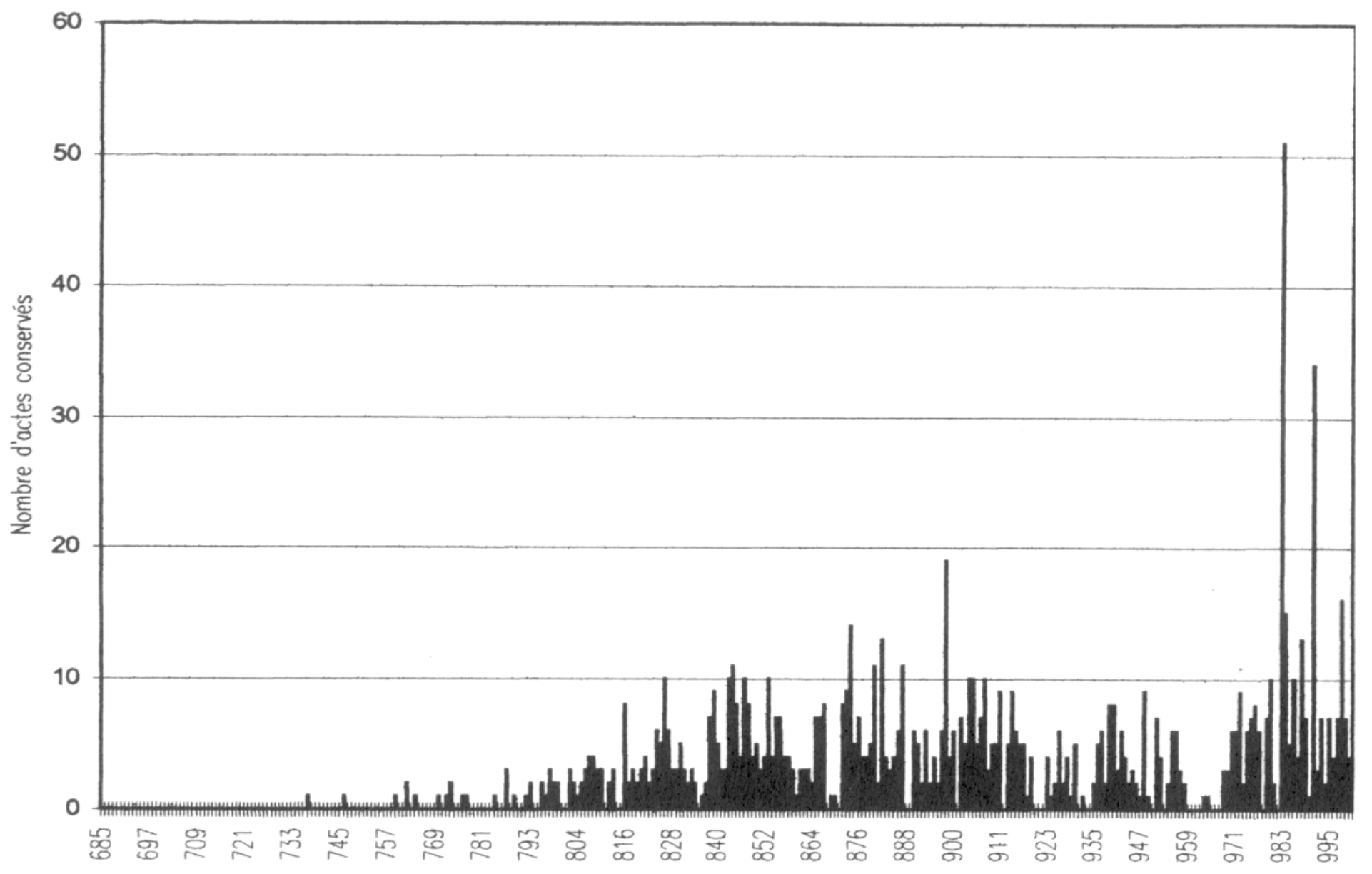

Le livello se distingue par sa définition et son statut ambivalents, à Lucques tout au moins. Il apparaît dans un premier temps comme tout type de concession à terme (sans que ce dernier soit d'ailleurs bien défini), comme un contrat agraire aussi bien que comme une concession de biens engageant un rapport de clientèle. Le terme "livellum" n'implique pas un lien juridique précis et univoque entre les contractants. En revanche, la jouissance des biens en usufruit moyennant différentes prestations (cens, travail) définies en fonction des liens qui unissent les contractants, est une constante. Le contrat agraire est caractérisé par certains engagements : de résidence, de soumission à la justice, de travail sur la pars dominica. Les concessions à cens sans autre clause contraignante font alors penser à des relations de clientèle, à une formalisation écrite des rapports entre l'évêque et l'aristocratie. Avant 780, le formulaire et la terminologie employés pour désigner ce rapport (convenentia et promissio) traduisent ce flottement juridique. Par la suite, la stabilisation du formulaire et l'emploi systématique du terme livellum n'impliquent pas pour autant une clarification : seule l'a- 
GRAPHIQUE $\mathrm{N}^{\circ} 6 \mathrm{~b}$. LIVELLI CONSERVÉS RÉPARTIS PAR PÉRIODES DE 20 ANS

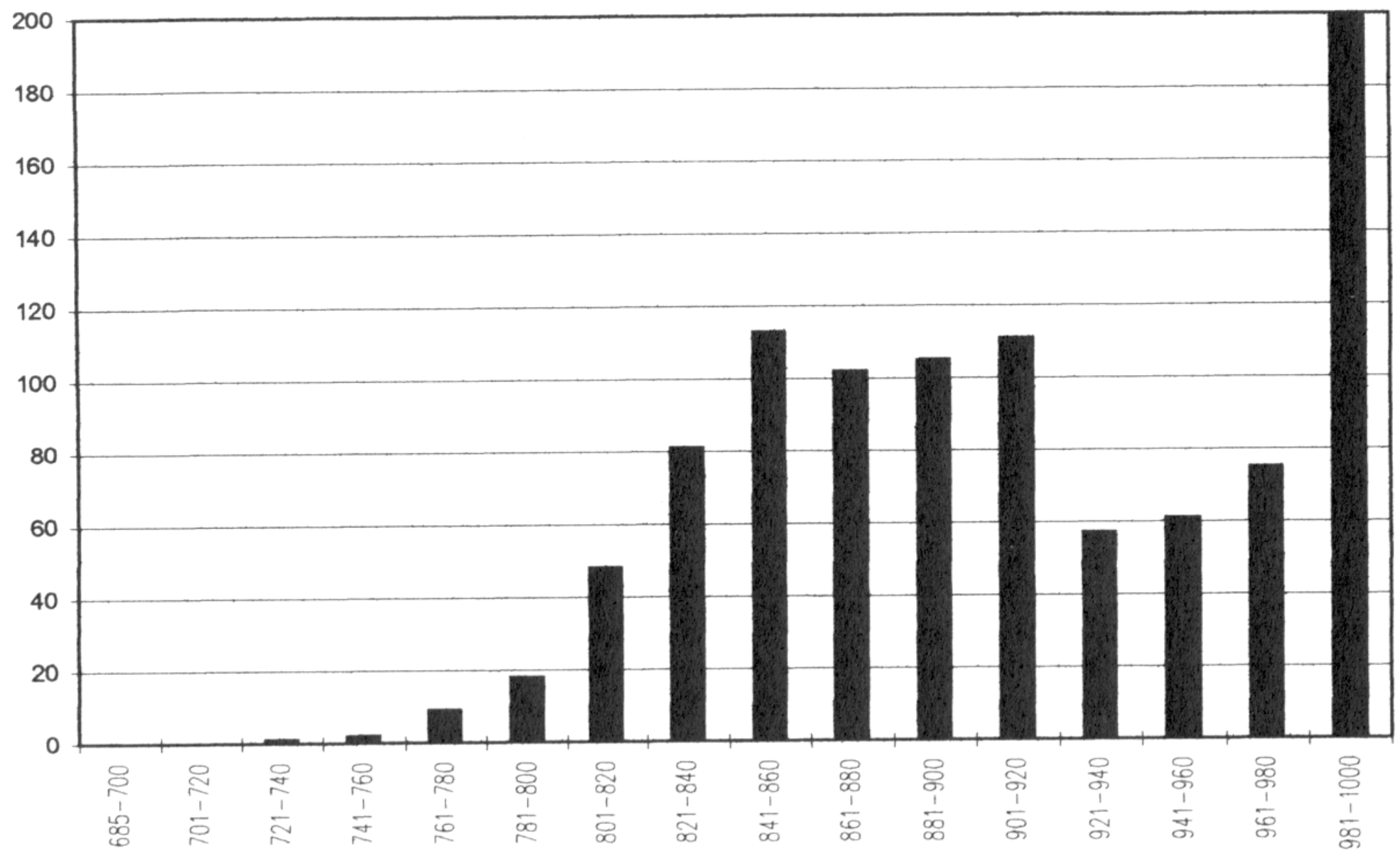

nalyse des clauses permet de les déterminer. On peut ainsi dater approximativement de 860 la disparition des contrats agraires établis directement par l'évêché. À partir de ce moment, les livelli apparaissent comme des concessions qui mettent exclusivement en jeu les évêques et leur entourage aristocratique. Parallèlement, l'apparition - et l'accroissement numérique - du livello au IX ${ }^{e}$ siècle peut être analysée comme le symptôme de l'entrée de l'évêché dans une phase de gestion de son patrimoine, et plus seulement de constitution et d'accroissement.

Quelques moments retiennent l'attention. Au début du IX siècle, quand les évêques appartiennent encore à l'aristocratie locale, la multiplication des livelli et des ordinations à la tête des ensembles ecclésiastiques placés sous la juridiction épiscopale (églises privées tombées dans son escarcelle, ou paroisses dotées d'un pouvoir territorial important) indique un repli patrimonial vers l'évêché : les Lucquois peuvent ainsi maintenir leur position sociale et limiter les effets de l'arrivée des Francs et de la confiscation des pouvoirs publics par les nouveaux cadres mis en place par 
Charlemagne et ses descendants à la tête du Regnum. En contrepartie, les évêques créent ou confortent ainsi leur assise locale et leur clientèle politique.

Le livello permet la consolidation de liens déjà mis en place au VIII ${ }^{c}$ siècle, comme c'est le cas pour Santa Maria Ursimanni, déjà citée, où la pratique concessionnaire prend le relais des donations réalisées par la famille d'Orso. Jusqu'au milieu du IX ${ }^{*}$ siècle, les relations entre la famille et l'évêché perdurent grâce aux livelli. Les ruptures politiques des années 820 ne perturbent pas totalement le lien privilégié qui unit cette institution et l'évêché : ainsi, au milieu du IX ${ }^{e}$ siècle, lorsque l'évêque Jérémie (Lucquois) prend ses fonctions, le contrat livellaire est renouvelé, après la rupture que représentent les pontificats de Bérenger et Ambroise.

Toutefois, le livello peut aussi être le moyen de recruter, pour ces nouveaux arrivants, une clientèle à leur service. Il convient ici de s'arrêter sur une anomalie dans la répartition chronologique des livelli : sous les pontificats de Bérenger et Ambroise, des pics correspondent probablement à l'annulation par ces deux hommes des livelli contractés par leurs prédécesseurs ${ }^{25}$ et à de nouvelles concessions en vue de composer un nouveau réseau de fidélité. Le livello permet à ces hommes "nouveaux» de parer à leur faible influence politique et à leur faible intégration locale. Puisque la concession formalisée par le livello n'est pas illimitée et qu'elle est susceptible d'être annulée (par le souverain dans le cas qui nous concerne), celuici est une arme formidable pour la formation ex nihilo d'une influence politique nouvelle, et parfois forcée. À la même époque, le livello devient réservé à des concessions non agraires : seuls l'évêque et son entourage aristocratique sont concernés. Plus que jamais, donc, le livello est un instrument politique. On le vit en 852 , lorsque Louis II installa à la tête de l'évêché Jérémie, d'origine lucquoise ${ }^{26}$, après le gouvernement de deux pontifes "étrangers». Peu de temps après, un précepte ordonnait l'annulation de tous les baux livellaires antérieurs à l'arrivée de Jérémie pour lui permettre de récupérer les biens concédés et d'améliorer la gestion du patrimoine épiscopal. En 853, un plaid montre l'évêque récupérant des terres cédées "abusivement" par son prédécesseur ${ }^{27}$; et la courbe des livelli, après une période de récession jusqu'en 852 , se caractérise par une reprise cette année-là ${ }^{28}$, où se conjuguent la nécessité de renouveler les contrats à cause de

${ }^{25}$ Cf. F. Bougard, La justice... et A. Mailloux, Pratiques notariales...

${ }^{26}$ Cf. H. Schwarzmaier, Lucca und das Reich..., p. 95 et suivantes. p. 189.

${ }^{27}$ Cf. AAL 721 (= C. Manaresi, I Placiti..., n" 57); F. Bougard, La justice...,

${ }^{28} \mathrm{Cf}$. graphiques n" 6 a et $b$. 
l'arrivée d'un nouvel évêque, mais aussi la situation nouvelle créée par l'annulation des baux précédents.

Toutefois, cette politique de concession n'est possible que parce que l'évêché vit sur des patrimoines acquis grâce aux donations de l'aristocratie laïque tout au long du VIII ${ }^{e}$ siècle. Paradoxalement, donc, les donationsrétrocessions imaginées par les Lucquois au début du $\mathrm{IX}^{\mathrm{e}}$ siècle ont servi essentiellement l'évêché, et en particulier les évêques, quelle que soit leur origine.

Le livello progresse dans sa forme et son contenu au cours du IX ${ }^{\mathrm{c}}$ siècle : le terme de la concession est peu précis (le plus souvent, il s'agit de contrats à deux, voire à trois générations) et les clauses du contrat mettent en évidence le fait qu'il s'agit de plus en plus de concessions à cens. Les obligations des preneurs sont limitées au paiement d'un cens peu contraignant. Sans être une aliénation, la concession représente pour l'évêché une perte de contrôle sur les parts de territoire et de biens ainsi laissées à son entourage laïque, compensée toutefois par la consolidation, par le même vecteur, de cette même clientèle. La situation peut devenir critique lorsque ce type de concession livellaire touche des biens fonciers attachés à des églises plébanes, qui sont concédées de la même façon que des biens fonciers à des laïcs.

Cette situation dangereuse atteint son point culminant à la fin du IX' siècle, où le nombre de livelli et leur proportion dans les transactions épiscopales conservées est écrasant ${ }^{29}$. Toutefois, un redressement de la gestion foncière de l'évêché est amorcé par l'évêque Pierre II, entre la fin du $\mathrm{IX}^{*}$ siècle et le début du $\mathrm{X}^{\mathrm{c}}$ siècle. À son entrée en fonction, Pierre II, d'origine lucquoise et issu d'une famille où l'on compte des notaires et scabins ${ }^{30}$ - donc rompu aux techniques juridiques et aux subtilités de la pratique contractuelle -, fait établir deux inventaires des biens de l'évêché, le premier portant sur ses biens fonciers, le second sur les concessions consenties à l'aristocratie laïque ${ }^{31}$. L'examen de ces documents montre qu'il existait un système de classement des actes de la pratique, que l'on peut retrouver aussi grâce aux mentions dorsales. Les pièces étaient probablement regroupées selon un critère topographique, repris dans les inventaires. Ceux-ci ont alors servi d'instrument à l'élaboration d'un diagnostic ou d'une expertise sur la structure du patrimoine épiscopal et ses dysfonctionnements. Le second inventaire (890-900) porte sur son verso, d'une

${ }^{29}$ Cf. supra note $24: 861-880: 66,6 \% ; 881-900: 72 \% ; 901-920: 77 \%$.

${ }^{30}$ Cf. H. Schwarzmaier, Lucca und das Reich..., p. 100-103.

${ }^{31} \mathrm{Cf}$. Inventari altomedievali di terre, coloni e redditi, Rome, 1979 (Fonti per la storia d'Italia, 104), n"s XI-1 et XI-2, éd. par M. Luzzatti. 
main du $\mathrm{X}^{\mathrm{e}}$ siècle, le titre "Breve de feora": il s'agissait de dresser la liste des concessionnaires, cette fois-ci bénéficiaires et livellaires, et de démêler l'écheveau complexe des relations contractées par les prédécesseurs de l'évêque. Il fallait consolider la propriété épiscopale et l'influence de ce dernier. Toutefois, au lieu de choisir la solution de l'annulation, comme c'était le cas de Jérémie quelque cinquante ans auparavant, l'évêque Pierre II se lance dans une opération lente de restructuration du domaine. Après quoi le nombre de livelli baisse, et l'on observe parallèlement la mise en place d'un système de concessions spécifiques destinées à réguler la gestion des églises plébanes : pour ces ensembles particuliers, Pierre II procède au rétablissement de concessions canoniques à des prêtres chargés de la cure d'âmes et liés, pour la gestion des biens fonciers, par des clauses similaires à celles que l'on peut rencontrer dans les livelli traditionnels, mais dont la durée est strictement limitée à la fonction pastorale du concessionnaire. On ne trouve pas, sous Pierre II, de livelli concédant des églises, des parts d'églises ou des dîmes à des laïcs.

Après cette baisse des livelli, traduisant dans un premier temps une réorganisation et une meilleure maîtrise du patrimoine, le nombre de concessions livellaires augmente à nouveau et témoigne alors d'une gestion raisonnée de ce patrimoine consolidé. Cette seconde phase de la gestion selon Pierre II correspond à une entreprise de reconcentration des efforts de l'évêché dans des zones particulièrement importantes du territoire. Certains livelli, concédés par groupes cohérents dans l'espace et dans le temps, rappellent une opération concertée d'incastellamento, à Santa Maria a Monte en particulier ${ }^{32}$. Le livello y est un outil souple et efficace, contraignant grâce aux multiples possibilités d'établir des clauses obligeant les concessionnaires à demeurer dans un lieu précis et à mettre en valeur, de façon indirecte le plus souvent, des portions précises du territoire ainsi remodelé, répondant en même temps à la nécessité pour l'évêché de conforter son assise sociale et politique. Cette politique équilibrée ne vaut toutefois que si l'évêque a conscience des dangers que représentent des livelli trop libéraux, ce qui semble être le cas pour Pierre II.

${ }^{32}$ Cette église, dépendant dans un premier temps de l'église plébane de Sant'Ippolito de Aniano, située dans la plaine, fait l'objet d'un soin particulier de la part de l'évêché, dès le IX $\mathrm{X}^{\mathrm{e}}$ siècle. En 898, sept contrats livellaires, délivrés par le prêtre recteur de la paroisse de $\mathrm{S}$. Ippolito et $\mathrm{S}$. Maria a Monte concède des parcelles à proximité de l'église Santa Maria, avec obligation d'y construire des bâtiments (dont la description indique clairement qu'ils sont compris dans un habitat groupé et entouré d'une muraille) et d'y demeurer, cf. B, V-2, nos 1008 à 1013, 1016. L'église Santa Maria a Monte n'est nommée plebs qu'en 983. 
Cette politique de réorganisation est rendue possible par une meilleure maîtrise des livelli, par la reprise en main par l'évêché de son patrimoine foncier (annulation éventuelle de livelli après la confection des inventaires, ou abandon des pratiques concessionnaires jusqu'à ce que le bien soit restitué à expiration du livello - mais cette dernière hypothèse n'est guère plausible, car il semble qu'à cette époque la concession livellaire connaisse la même évolution que le bénéfice carolingien vers la transmission héréditaire) et surtout par le maintien d'un volant foncier, voire par l'accroissement du patrimoine grâce à la pratique des échànges, qui prennent à la même époque une tournure particulière et l'allure de donations déguisées.

\section{LES ECHANGES}

L'échange, appelé dans la documentation lucquoise viganeum ou cambiatio, implique un accord et une parité des parties. Il doit théoriquement se faire à valeur égale des biens, ce qui implique des opérations d'évaluation et de bornage des terres. Son "égalité» est validée par la présence, obligatoire et respectée, d'un scabin, ou d'un missus par la suite ${ }^{33}$, et concrétisée par la rédaction en double d'un acte de même teneur mais comportant un formulaire différent pour chacune des parties. Les échanges portent sauf exception sur des biens fonciers ${ }^{34}$. En valeur absolue, leur nombre est assez régulier sur l'ensemble de la période considérée, ce qui laisse penser qu'on y recourt de manière fréquente. Seules deux baisses sont perceptibles, entre 780 et 800 et de 901 à $920^{35}$.

La première baisse s'explique par deux facteurs conjoints : le marché foncier fondé sur les ventes est alors en expansion, tandis que la diversité typologique des actes conservés s'élargit. Les ventes et les donations prévalent sur ce type de mobilité foncière mineur. D'autre part, les échanges de la première période (pendant le VIII ${ }^{\mathrm{e}}$ siècle et la première moitié du $\mathrm{IX}^{\mathrm{e}}$ siècle) se font réellement à parité. De plus, ils engagent des biens de taille limitée, réorganisés dans un souci de concentration parcellaire, essentiellement dans la plaine de Lucques, où la propriété est très morcelée. Ces échanges de parcelle à parcelle servent alors essentiellement au re-

${ }^{33}$ Cf. A. Mailloux, Perception de l'espace chez les notaires de Lucques, dans MEFRM, 109, 1997, p. 21-57, spéc. p. 45. Voir aussi F. Bougard, La justice..., p. 151 et 178-181. Une représentation publique est prévue dans la loi lombarde (Aistulf 16). Les missi présents aux $\mathrm{IX}^{\mathrm{e}}$ et $\mathrm{X}^{\mathrm{e}}$ siècles sont soit ducaux soit royaux.

${ }^{34}$ Un seul acte témoigne de la possibilité d'échanger des hommes (servi et ancillae), AAL 100 (B, V-2, no 98, de 766).

${ }^{35} \mathrm{Cf}$. graphiques $\mathrm{n}^{\circ} 7$ a et $\mathrm{b}$, "Répartition chronologique des échanges». 
GRAPHIQUE N`7a. RÉPARTITION CHRONOLOGIQUE DES ÉCHANGES

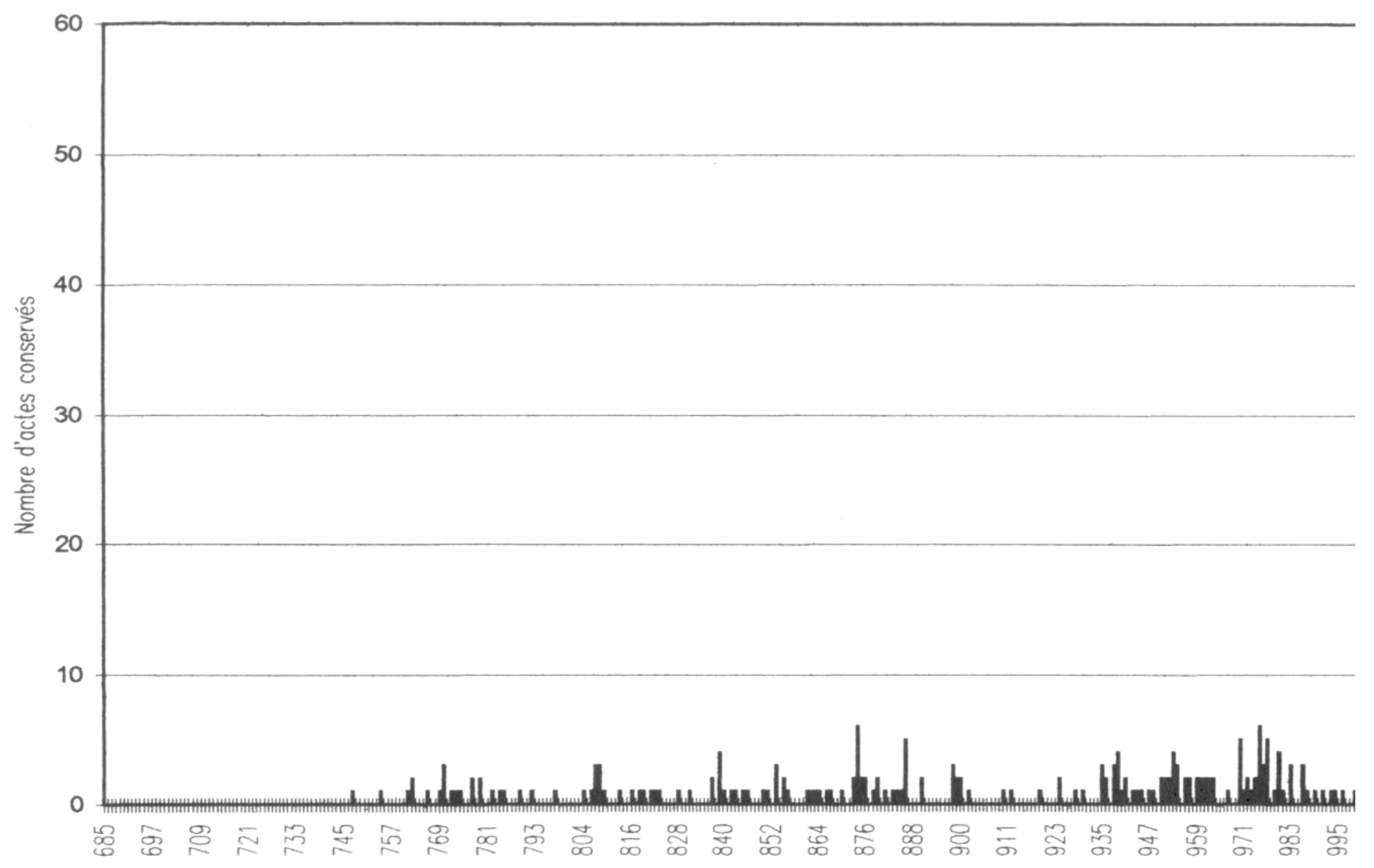

membrement des terres, à une échelle restreinte. On peut les considérer comme une pratique de gestion, plus que comme un outil d'agrandissement du patrimoine.

En revanche, la seconde baisse des échanges, qui s'observe au moment de la réorganisation de l'évêque Pierre II, rend compte d'évolutions plus complexes. Les échanges de biens sont alors à leur étiage (seulement 2,7\% des actes), alors qu'ils représentent une part notable des transactions entre 861 et $900(14,3$ et 12,5\%), part qui s'accroît suite (18\% de 921 à 940 et $25 \%$ de 941 à 960). Une remarque liminaire s'impose : les échanges ont changé de teneur entre le VIII ${ }^{\mathrm{e}}$ siècle et la fin du IX ${ }^{\mathrm{e}}$ siècle. La fiction de l'échange à parité est maintenue, la présence des missi est toujours requise, de même que les opérations de bornage et d'évaluation des parcelles échangées. Toutefois, la clause de melioratio en faveur de l'église est de plus en plus fréquente. Elle apparaît dans le courant du IX ${ }^{c}$ siècle et se généralise au $\mathrm{X}^{\mathrm{e}}$ siècle. Elle consiste à "améliorer" la part qui échoit à l'évêché : la description des biens échangés met en évidence le fait que les parcelles don- 
GRAPHIQUE N ${ }^{\circ}$ b. ECHANGES CONSERVÉS PAR PÉRIODE DE 20 ANS

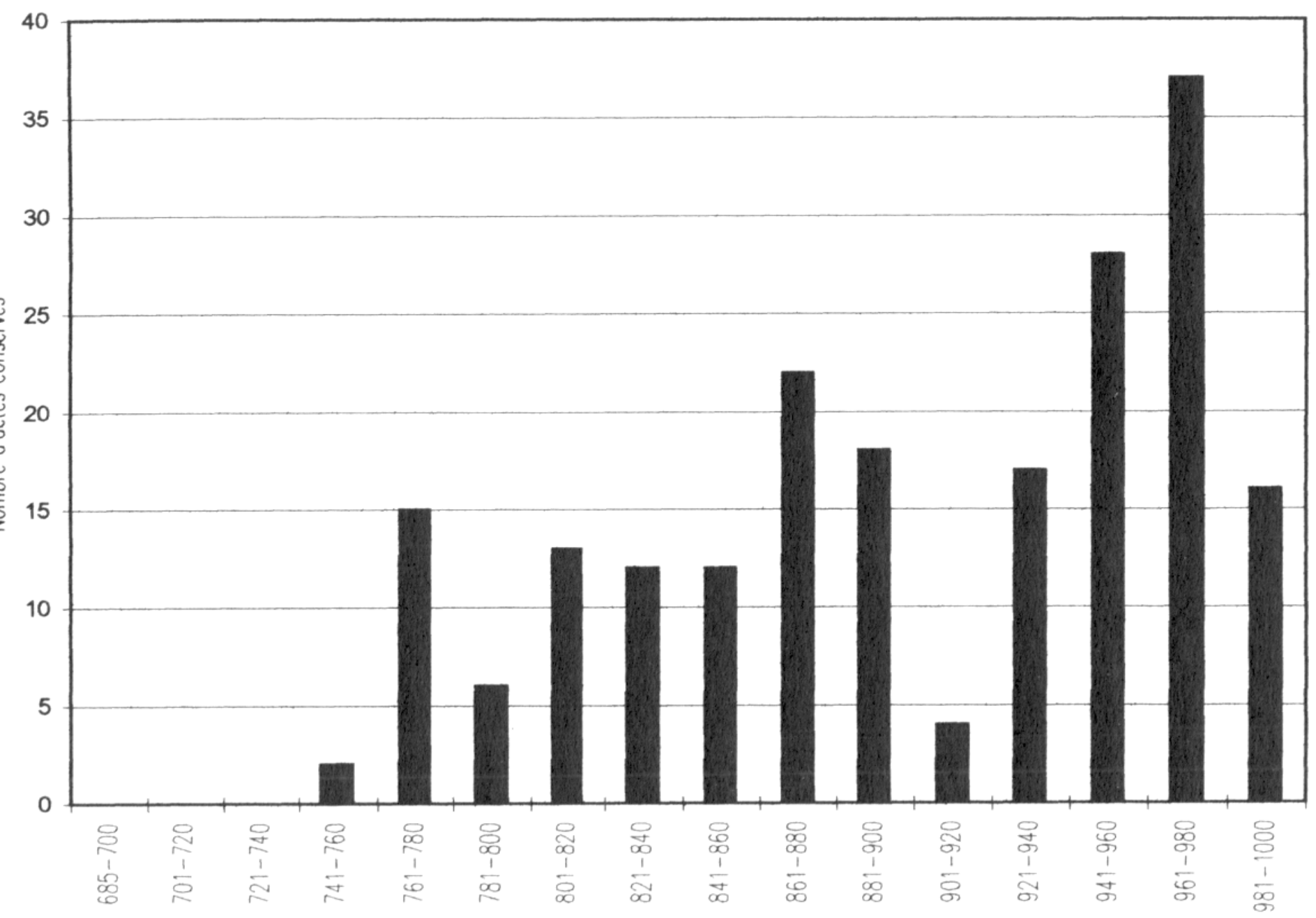

nées à l'église sont plus nombreuses et plus étendues que celles qu'elle veut bien céder. Autre différence par rapport aux échanges de la première phase, les surfaces en jeu sont plus étendues, des pans entiers de patrimoine changent de propriétaire, parfois disséminés sur l'ensemble du diocèse de Lucques. Enfin, la situation des biens échangés est révélatrice d'un mouvement nouveau : si dans la première période, les échanges de parcelle à parcelle se font dans une même zone, voire dans un même terroir ${ }^{36}$, ils sont réalisés au cours du $\mathrm{X}^{*}$ siècle sur des ensembles très distants les uns

${ }^{36} \mathrm{Cf}$. A. Mailloux, Pour une étude des paysages dans le territoire de Lucques au haut Moyen Âge (VIII' siècle), dans De la terre au ciel. I. Paysages et cadastres antiques (XII stage international, Besançon, 29-31 mars 1993), Besançon, 1994, p. 207-222. Les échanges ici rappelés ont lieu dans la plaine de Lucques, et dans la zone de Lunata en particulier. 
des autres. En général, les propriétaires laïcs donnent des biens dispersés sur tout le diocèse, et reçoivent de l'évêque des biens groupés, formant le plus souvent un ensemble cohérent, décrit de façon caractéristique ${ }^{37}$, mais de surface moindre. La melioratio a donc bien eu lieu, mais on ne conçoit pas immédiatement l'intérêt des donateurs laïcs à procéder à ce type d'opération.

Nous entrons là dans un jeu social subtil : dans un marché foncier bloqué (pour ce que l'on peut en percevoir), l'échange est le seul moyen d'agir sur les patrimoines et leur agencement. Il représente pour les laïcs une perte de terres, puisque la plupart du temps l'échange est aussi une donation cachée, mais il est en même temps le seul moyen de regrouper les propriétés et de procéder à un réaménagement du territoire aboutissant à la construction de châteaux. Pour l'évêché, l'avantage est d'abord, grâce à la melioratio, d'acquérir des propriétés sans bourse délier, mais il est aussi de renforcer une clientèle souvent volatile à cause des modifications des rapports induits par l'évolution du livello. L'évêque fait de ses interlocuteurs ses obligés : il leur donne en échange de patrimoines épars des ensembles plus petits mais bien plus cohérents territorialement. C'est pour les laïcs un bon moyen de constituer de petites unités territoriales où implanter un castrum sur un espace tenu en pleine propriété (avantage juridique que ne donne pas un livello).

La seconde baisse proportionnelle des échanges, sous l'autorité de Pierre II, semble ainsi répondre à des raisons plus claires : l'œuvre de Pierre II consiste en une restructuration et une reprise en main du patrimoine ecclésiastique, mais aussi de la maîtrise territoriale (meilleur contrôle sur les plebes, formation d'ensembles châtelains cohérents et commandant le territoire du diocèse grâce à leur position stratégique, aux points de contrôle de l'Arno - Santa Maria a Monte - et de la plaine de Lucques - Moriano). Ralentir le rythme des échanges est une mesure qui accompagne le gel des livelli : on met un frein à une dispersion accrue du

${ }^{37}$ Cf. entre autres un échange du 3 octobre 935 (B, V-3, $\mathrm{n}^{\circ} 1233$ ), intervenant entre l'évêque Conrad et deux frères, Auderam et Ildibald. Les frères donnent 26 parcelles, couvrant 31 muids et 225 setiers, contre 4 parcelles de 14 muids et 58 setiers. La description d'un des ensembles reçus par les deux frères est la suivante : «Recepi (...) colle illo in loco et finibus Massa, ubi dicitur Coterocto prope eccl. S. Cristine, pertinentes suprascripte eccl. epis. vestro S. Martini, et est tenentes ipsa terra et colle uno capo cum uno lato in via publica, alio capo cum alio lato tenet in terra suprascripte eccl. epis. vestro (...). Has suprascriptas terra et colle, qualiter circundata est per designatas locas, quod est per mensura ad justa perticha mensuratas mod. nove in integrum, una cum accessionibus et ingressoras suas, seo cum inferioribus et superioribus ad te eas in commutationem recepi (...)". 
patrimoine épiscopal, à un moment où l'incastellamento imposait de concentrer, et à une perte de contrôle juridique sur les ensembles échangés. Les échanges, dont le rythme rythme est lié à celui des livelli, sont le facteur équilibrant des relations entre les évêques et leurs interlocuteurs aristocratiques.

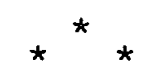

La cohérence exceptionnelle du fonds épiscopal de Lucques permet de suivre au plus près les dynamiques patrimoniales de cette institution; surtout, le caractère continu de la documentation donne la possibilité non seulement de les décrire, mais aussi de les analyser dans leur contexte social et politique. Le VIII e siècle voit la formation et l'accroissement du patrimoine, le IX ${ }^{e}$ siècle la gestion, fondée essentiellement sur la pratique concessionnaire. Le bouleversement perceptible au début du $\mathrm{X}^{\mathrm{e}}$ siècle engage une modification formelle des rapports sociaux et politiques locaux et territoriaux. Le livello-échange se substitue à la donation-rétrocession : sous cette forme différente, une action plus précise, plus directe sur la forme du territoire est favorisée par le transfert de pans entiers de la propriété, par une redistribution massive qui affecte l'ensemble des terres épiscopales. L'évêché y trouve un moyen d'accroître ses biens, même s'ils sont plus diffus. Toutefois, cette dispersion d'apparence n'induit pas de perte de contrôle, car l'évêché a procédé dans une première phase à des opérations d'incastellamento qui lui ont permis de contrôler les points essentiels du territoire. Les pôles seigneuriaux "enchâtelés" qui se créent à partir de 920 sous l'égide et le contrôle de l'évêché ne nuisent pas à la mainmise de l'évêque sur cet espace. 